Research Article

\title{
De novo transcriptome analysis of Tibetan medicinal plant Dysphania schraderiana
}

\author{
Suhong $\mathrm{Fu}^{1 *}$, Ming Lei ${ }^{2 *}$, Yongqun Zhang ${ }^{1}$ iD, Zhaomin Deng ${ }^{1}$, Jing $\mathrm{Shi}^{1}$ and Doudou Hao ${ }^{1}$ \\ ${ }^{1}$ Molecular Medical Laboratory, Hospital of Chengdu Office of People's Government of Tibetan \\ Autonomous Region, Chengdu, China. \\ ${ }^{2}$ School of Science, Tibet University, Lhasa, China.
}

\begin{abstract}
Dysphania schraderiana is widely distributed in Lhasa (Tibet, China) and used as a traditional medicine. However, the lack of genetic information hinders the understanding of its physiological processes, such as the biosynthesis of secondary metabolites. Herein, we used Illumina Hiseq 4000 platform to sequence the transcriptome of flower and leaf tissues from $D$. schraderiana for the first time. Totally, 40,142 unigenes were assembled from approximately 5.2 million clean reads. All unigenes underwent gene prediction and were subsequently annotated in a NR (NCBI non-redundant protein) database, COG (Clusters of Orthologous Groups of proteins) database, and KEGG (Kyoto Encyclopedia of Genes and Genomes) database. Among the 40,142 unigenes, 2,579 genes were identified as differentially expressed between flowers and leaves, and used in further enrichment analysis. Also, 2,156 unigenes were annotated as transcription factors. Furthermore, our transcriptome analysis resulted in the identification of candidate unigenes annotated to enzymes involved in terpenoid biosynthesis. Taken together, this work has laid the foundation for the investigation of secondary metabolite biosynthesis and other physiological processes of $D$. schraderiana.
\end{abstract}

Keywords: Dysphania schraderiana, de novo assembly, transcriptome, annotation, terpenoid biosynthesis.

Received: February 08, 2018; Accepted: October 16, 2018.

\section{Introduction}

Dysphania schraderiana, in the Chenopodiaceae family, is widely distributed in the Qinghai-Tibet Plateau of China, Europe, and Africa. The Qinghai-Tibet Plateau is a low oxygen, low temperature, strong ultraviolet radiation, and poor soil environment. The adaptation of $D$. schraderiana to these extreme environments can be a good model for understanding evolutionary ecology. Besides, $D$. schraderiana was used as an indicator of fixed sand because of its high adaption to environmental adversities (Liu, 2013). Apart from its ecological value, the plant is used in a variety of applications such as medicine and insect control. In traditional Chinese medicine, D. schraderiana is used to, amongst others, mitigate wheeze, inflammation, spasm, migraine (Xie, 1996). Some recent studies indicated that the essential oil of $D$. schraderiana could be applied to prevent plant mite (Liu, 2015). In addition, the essential oil of $D$. schraderiana also has in vitro antibacterial activity against Escherichia coli, as well as anti-insect activity against red flour beetle and corn weevil (Lei et al., 2015a,b;

Send correspondence to Yongqun Zhang. Molecular Medical Laboratory, Hospital of Chengdu Office of People's Government of Tibetan Autonomous Region, 610041 Chengdu, China. E-mail: yongqunzhang@yahoo.com.

"These authors contributed equally to this work.
Shi, 2015). These findings suggest that D. schraderiana can became a promising antibacterial agent considering the increasing episodes of drug resistant bacteria. Moreover, the essential oil can also be utilized in foods, cosmetics, and cigarettes (Han et al., 2013). Despite the importance of $D$. schraderiana and some genomic research on Chenopodiaceae plants such as Beta vulgaris ssp. vulgaris, and Chenopodium quinoa (Chou et al., 2017; Zhang et al., 2017), no genetic information exists for this non-model genus. Therefore, it is necessary to explore genetic data sources of $D$. schraderiana for gene discovery and further functional studies.

In essential oils of $D$. schraderiana, 52 phytochemical compounds were identified by GC-MS (Finnigan Voyager) analysis and the dominant components are sesquiterpenes and oxygen-containing derivatives, followed by monoterpenes (Shi, 2015). The synthesis of terpenoids relies on two key pathways, the 2-C-methyl-derythritol-4phosphate (MEP) and mevalonate (MVA) pathways, involving diverse enzymes (Goodwin, 1979; Rohdich et al., 2001; Cheng et al., 2007). Isopentenyl pyrophosphate (IPP) and dimethylallyl pyrophosphate (DMAPP), the universal precursors for terpenoids, are synthesized through the MEP and MVA pathways (Rohdich et al., 2001). GPP synthase catalyzes IPP and DMAPP to form geranyl diphosphate 
(GPP), while FPP synthase converts GPP into farnesyl diphosphate (FPP) (Rohdich et al., 2001). GPP and FPP are catalyzed to form monoterpenes and sesquiterpenes by monoterpene synthase (mono-TPS) and sesquiterpene synthase (sesqui-TPS), respectively (Gershenzon, 2009). The discovery and research of key enzymes related to terpenoid biosynthesis in D. schraderiana could help understand the composition of the essential oil.

Nowadays, de novo assembly is gaining more attention and has proved to be a rapid and cost-effective method for short reads in non-model organisms (Crawford et al., 2010). Millions of short tags could be generated from RNA-Seq platforms and subsequently assembled, which can help to interpret genome and transcriptome sequences. Collectively, D. schraderiana could be a valuable source to identify and discover natural compounds with biological activity for further pharmaceutical research, and be used to study the adaptive genetic mechanism. In this study, we characterized the flower and leaf transcriptomes of $D$. schraderiana using Illumina sequencing platform, and annotated sequences from transcriptome in multiple databases.

\section{Materials and Methods}

\section{Plant materials}

Whole flowers and young leaves of D. schraderiana were collected from the new campus of Tibet University (N29 $\left.38^{\prime}, \mathrm{E}^{\circ} 1^{\circ} 10^{\prime}\right)$, Lhasa, Tibet. Plant materials were quickly frozen in liquid nitrogen upon harvest and stored at $-80{ }^{\circ} \mathrm{C}$ until RNA extraction.

\section{RNA extraction and quality determination}

Total RNA was isolated using Trizol (Invitrogen Inc, USA). RNA quantity and quality was checked using a Nanodrop2000 spectrophotometer. Agarose gels (1\%) were utilized to monitor RNA degradation and contamination. RNA integrity was assessed using an Agilent2100 system (Agilent Technologies, CA, USA) at OD 260/280-1.8 to $2.2, \operatorname{RIN} \geq 8,>12 \mu \mathrm{g}$.

\section{cDNA library construction for Illumina sequencing}

The TruseqTM RNA Sample Prep Kit (Illumina, USA) was used to prepare the RNA-seq transcriptome libraries. DNA-free mRNA was captured by magnetic Oligo (dT) beads (Invitrogen) and fragmented to a size of $200 \mathrm{bp}$ with a fragmentation buffer. Using mRNA as a template, double-stranded cDNA was synthesized with a SuperScript double-stranded cDNA Synthesis Kit (Invitrogen) using random hexamer primers (Illumina). Then the end fragments were subjected to end-repair and ' $\mathrm{A}$ ' base addition. PCR amplification was carried out for 15 PCR cycles. Then, cDNA target fragments were selected on $2 \%$ Certified Low Range Ultra Agarose (Bio-Rad, USA) and quantitated by TBS380 Picogreen (Invitrogen).
The clustering of the index-coded samples was performed on a cBot Cluster Generation System using the TruSeq PE Cluster Kit v3-cBot-HS (Illumina) according to the manufacturer's instructions. After cluster generation, the library preparations were sequenced on an Illumina Hiseq4000 Truseq SBS Kit v3-HS (200 cycles).

\section{De novo assembly and sequence annotation}

Raw reads were cleaned by removing: adapters that were added for reverse transcription and sequencing; reads containing $\mathrm{N}$ (unknown nucleotides) more than $10 \%$; low quality reads with average phred scores less than 20 ; and sequences of less than $20 \mathrm{bp}$ after trimming, which was performed with SeqPrep (https://github.com/jstjohn/SeqPres) and Sickle (https://github.com/najoshi/sickle). The Q20, Q30, GC content, and sequence duplication level of the clean data were calculated (Erlich et al., 2008). All clean reads were used for de novo assembly into unigenes using Trinity (http://trinityrnaseq.sourceforge.net/) (Cock et al., 2009; Grabherr et al., 2011). Results of assembled unigenes were used to gather statistics from the basic indicators.

All the assembled unigenes of the two tissues were submitted to gene prediction using the ORF prediction procedure implemented in Trinity, then corrected against the Pfam database (http://pfam.sanger.ac.uk/). Finally, they were aligned using BLASTX (Version 2.2.25) (Camacho et al., 2009) against the Non-redundant (Nr, ftp://ftp.ncbi.nih.gov/blast/db/FASTA/nr.gz) (E-value threshold of 1.00e-5) (Deng et al., 2006), the String database, the Swissprot database, the Clusters of Orthologous Groups of proteins (Harris, 2004; COG, http://www.ncbi.nlm.nih.gov/COG/), and the Kyoto Encyclopedia of Genes and Genomes (KEGG, http://www.genome.jp/kegg/) (Kanehisa et al., 2004) database to acquire the corresponding annotation information.

\section{Identification and functional characterization of differentially expressed genes}

To investigate the expression profiles of unigenes from flower and leaf tissues of $D$. schraderiana, highquality reads from each sample were mapped on the Trinity transcripts assembly using Bowtie (http://bowtiebio.sourceforge.net/) (Langmead et al., 2012). RESM (http://deweylab.biostat.wisc.edu/rsem/) was used to filter and count the mapped reads (Li et al., 2011). Differential expression values were computed with edgeR (version 2.12) (Robinson et al., 2010), and gene expression levels were presented as FPKM (fragments per kilobase of exon per million fragments mapped) score (Mortazavi et al., 2008). Differentially expressed genes were considered significant at a false discovery rate $(\mathrm{FDR})<0.05$, adjusted value of 0.05 (Benjamini and Hochberg, 1995), and $\mid \log 2$ $\mathrm{FC} \mid \geq 1$ (FC, posterior fold change). The identified DEGs were used for GO and KEGG enrichment analysis, performed using Goatools (Young et al., 2010) 
(https://github.com/tanghaibao/GOatools) and KOBAS (http://kobas.cbi.pku.edu.cn/home.do) (Xie et al., 2011) respectively.

\section{Identification of transcription factors}

Transcription factors (TFs) were identified by analyzing InterProScan domain patterns in protein sequences with high coverage and sensitivity using the PlantTFcat analysis tool (http://plantgrn.noble.org/PlantTFcat/) (Dai et al., 2013).

\section{Results}

Transcriptome sequencing and de novo transcriptome assembly

cDNA samples from leaves and flowers of $D$. schraderiana were sequenced using Illumina sequencing. In total, 28,589,570 raw reads from leaves and 26,126,142 raw reads from flowers were generated. The high-quality raw data was deposited in the NCBI SRA database with accession number SRX3145241 and SRX3145242. The quality of reads is reported in Table S1, including Error, Q20\%, $\mathrm{Q} 30 \%$, and GC\%. Using the Trinity program, all clean reads were further de novo assembled into 48,908 transcripts with an average length of $873.13 \mathrm{bp}$ and an N50 of
$1450 \mathrm{bp}$ (Table 1). Then, a total of 40,412 unigenes were generated with the clustering and assembly analysis of transcripts. The length distribution of unigenes is illustrated in Figure 1, indicating the highest concentration in the 1-400 and $>800$ length range.

\section{Functional annotation and classification}

The results of the functional annotation showed that $23,864(59.45 \%)$ of the 40,142 unigenes were annotated against the Nr, Pfam, String, Swissprot, COG, and KEGG databases. As results, 23,723 (59.10\%) unigenes had significant marches in the $\mathrm{Nr}$ database, $14,572(36.30 \%)$ in the Pfam database, 11,843 (29.50\%) in String, 12,978 $(32.33 \%)$ in Swissprot, 9,950 (24.79\%) in KEGG, and $5,921(15.37 \%$ ) in the COG database (Table 2).

Of the annotated sequences in the non-redundant $(\mathrm{Nr})$ protein database, $72.2 \%$ displayed significant homology (E value $<1 \mathrm{E}-30$ ). The E-value distribution is shown in Figure $2 \mathrm{~A}$ and as shown in Figure 2B, the BLAST search analysis further revealed that a total of 3,300 (13.91\%) unigenes had

Table 1 - Summary of sequencing data and de novo assembling.

\begin{tabular}{lccccc}
\hline & Total number & Mean Length & GC $\%$ & N50 & N90 \\
\hline Transcripts & 48908 & 873.13 & $42.07 \%$ & 1450 & 338 \\
Unigenes & 40142 & 809.78 & $42.22 \%$ & 1425 & 300 \\
\hline
\end{tabular}

\section{Seq Length Distribution}

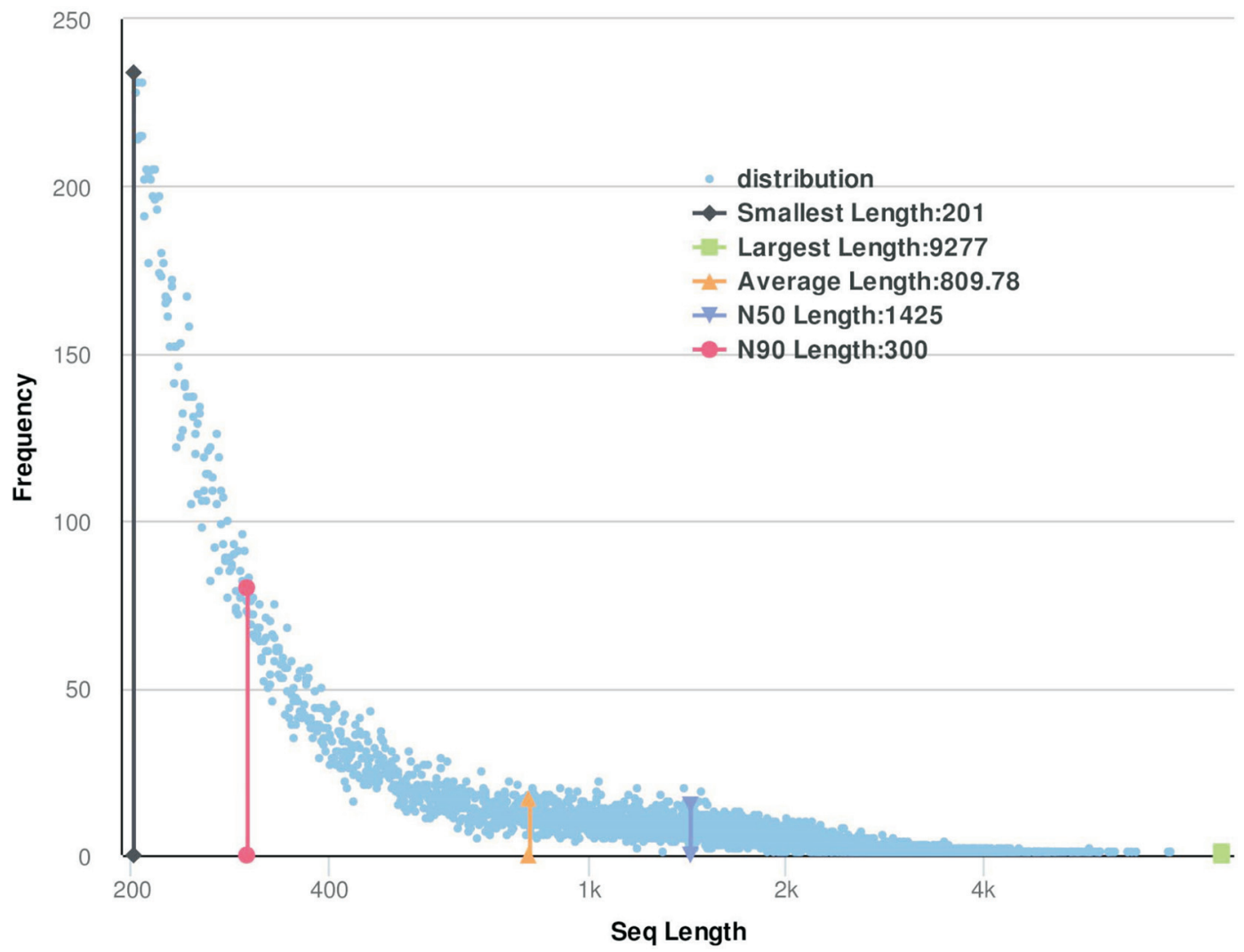

Figure 1 - Sequences length distribution. 
Table 2 - Summary of functional annotations for unigenes of $D$. schraderiana.

\begin{tabular}{lcc}
\hline Annotated Database & Unigenes & Frequency \\
\hline All Annotation & 23,864 & $59.45 \%$ \\
$\mathrm{Nr}$ & 23,723 & $59.10 \%$ \\
Pfam & 14573 & $36.30 \%$ \\
String & 11,842 & $29.50 \%$ \\
Swissprot & 12,979 & $32.33 \%$ \\
KEGG & 9,950 & $24.79 \%$ \\
COG & 6168 & $15.37 \%$ \\
\hline
\end{tabular}
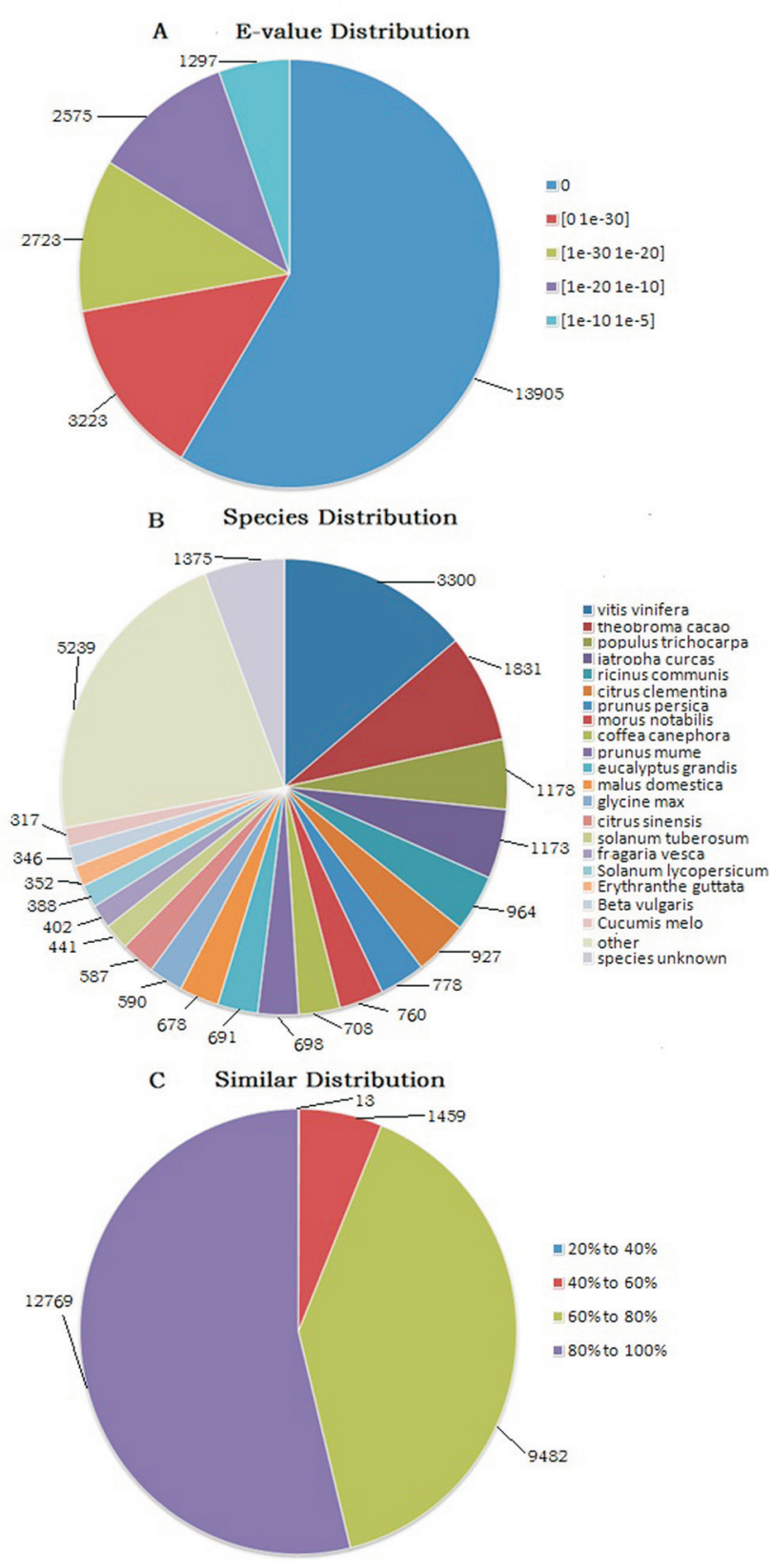

Figure 2 - Species distribution of unigenes from D. schraderiana. (A) E-value distribution of BLAST hits for each unigene with a cut off E-value of 1.0E-5. (B) Species distribution of top BLAST hits in the $\mathrm{Nr}$ database. (C) Similarity distribution of top BLAST hits for each unigene. the most similar sequences to proteins from Vitis vinifera, followed by Theobroma cacao 1,831 (7.72\%), and Populus trichocarpa 1,178 (4.97\%). In Figure 2C, the distribution analysis showed that $53.83 \%$ unigenes had a similarity of more than $80 \%, 39.97 \%$ unigenes had a similarity between 60 and $80 \%$, and $6.2 \%$ unigenes had a similarity of less than $60 \%$.

All the assembled unigenes were searched against the COG database and classified in clusters of orthologs. Overall, 6,168 unigenes were assigned 25 COG functional classifications with term abbreviations ranging from $\mathrm{A}$ to $\mathrm{Z}$ (except X). The largest category was the General Function prediction $(882,14.30 \%)$; the second category was Signal Transduction mechanisms (746, 12.09\%); and the third categories were Post-translational modification, Protein turnover, and Chaperon $(667,10.81 \%)$ (Figure 3). Only few unigenes were assigned to Cell motility and Nuclear structure, and no unigene was assigned to the Extracellular structures category.

The KEGG analysis showed that 9,950 unigenes were assigned to 341 KEGG pathways (Table S2), and these were classified into five larger pathway categories: metabolism (6854), genetic information processing $(2,136)$, environmental information processing (1102), cellular processes (1143), and organism system (1,903) (Figure S1). The top 20 KEGG pathways that contained transcripts are shown in Figure 4 . The most highly represented pathway was the Metabolic pathway (2,994 transcripts), followed by Biosynthesis of secondary metabolites (1,417 transcripts).

\section{Identification and functional characterization of differentially expressed genes (DEGs)}

The expression levels of 40,142 unigenes from flower and leaf tissue samples were represented by FPKM scores. As a result, a comparison between the flower and leaf tissue groups resulted in 2,579 significantly ( $p$-value $<0.05$ ) DEGs with $|\log 2 \mathrm{FC}| \geq 1$ and FDR $<0.05$ (Table S3). Statistical analysis of tissue-specifically expressed unigenes indicated that 234 and 146 unigenes were specific to flower and leaf tissues separately. Deep functional studies of these tissue-specific unigenes might provide additional insights into plant development. In addition, 2,199 DEGs commonly exist in both flower and leaf tissues (Figure $5 \mathrm{~A}$ ). In total, 1,476 unigenes exhibited up-regulation and 1,103 unigenes appeared down-regulated in leaves compared to flowers, among these 2,579 DEGs (Figure 5B). The DEGs were further analyzed using the KEGG database to explore their functional categories (Table S4). Among the 2,579 DEGs, 159 were annotated to phenylpropanoid biosynthesis, followed by starch and sucrose metabolism (113), phenylalanine metabolism (106), and carbon metabolism (105).

The GO enrichment analysis and KEGG enrichment analysis of DEGs of $D$. schraderiana was utilized to elucidate functional differences between flower and leaf tissues. 


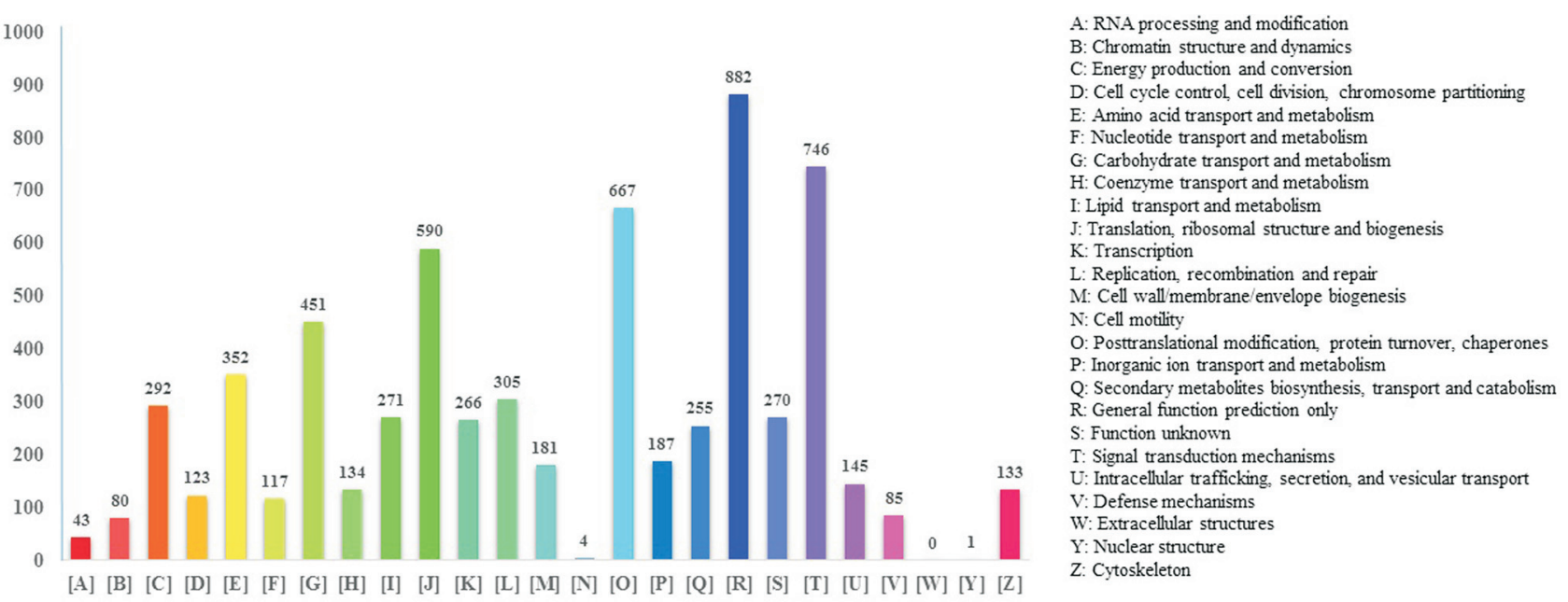

Figure 3 - COG functional classification of unigenes of $D$. schraderiana.

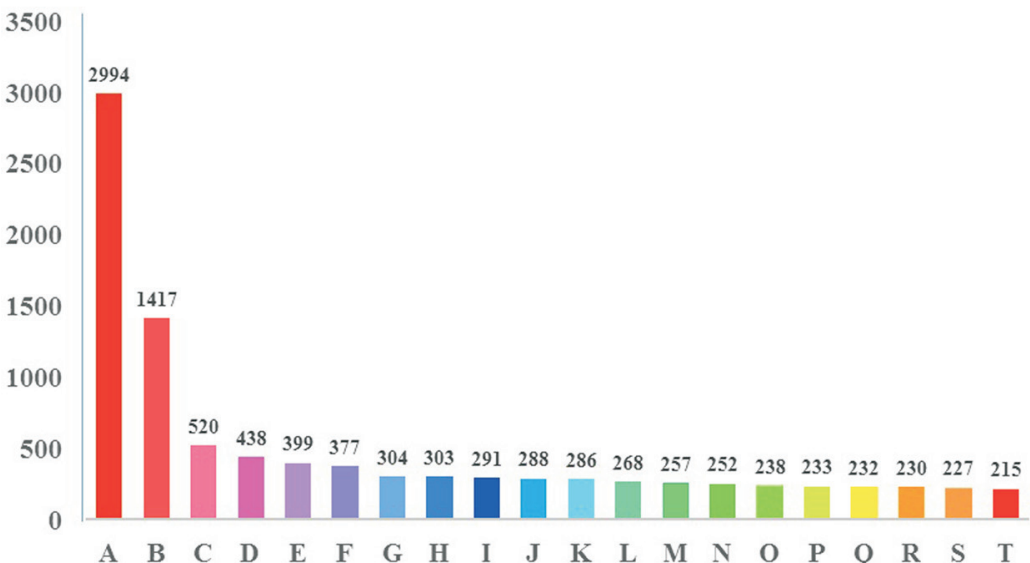

A: Metabolic pathways

B: Biosynthesis of secondary metabolites

C: Microbial metabolism in diverse environments

D: Ribosome

E: Carbon metabolism

$\mathrm{F}$ : Biosynthesis of amino acids

$\mathrm{G}$ : Protein processing in endoplasmic reticulum

H: Spliceosome

I: RNA transport

$\mathrm{J}$ : Starch and sucrose metabolism

$\mathrm{K}$ : Oxidative phosphorylation

L: Purine metabolism

M: Huntington's disease

$\mathrm{N}$ : Plant hormone signal transduction

O: Alzheimer's disease

$P$ : Epstein-Barr virus infection

Q: Endocytosis

$\mathrm{R}$ : Phenylpropanoid biosynthesis

$\mathrm{S}$ : Plant-pathogen interaction

$\begin{array}{llllllllllllllllllll}\text { A } & \text { B } & \text { C } & \text { D } & \text { E } & \text { F } & \text { G } & \text { H } & \text { I } & \text { J } & \text { K } & \text { L } & \text { M } & \text { N } & \text { O } & \text { P } & \text { Q } & \text { R } & \text { S } & \text { T }\end{array}$

T: Parkinson's disease

Figure 4 - Top 20 KEGG pathways assigned to the assembled transcripts.

A

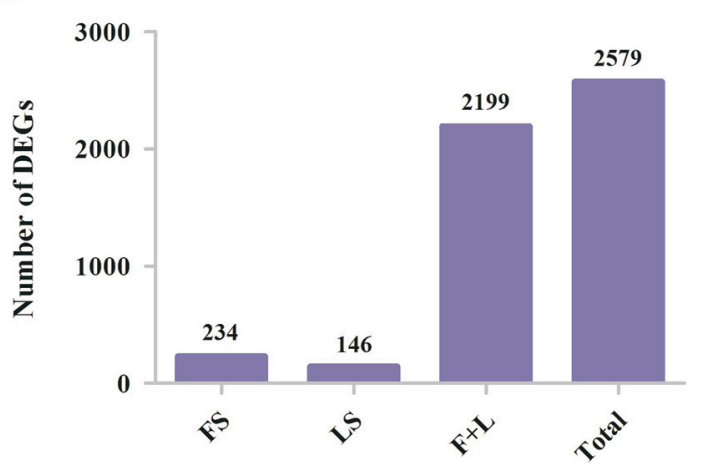

B

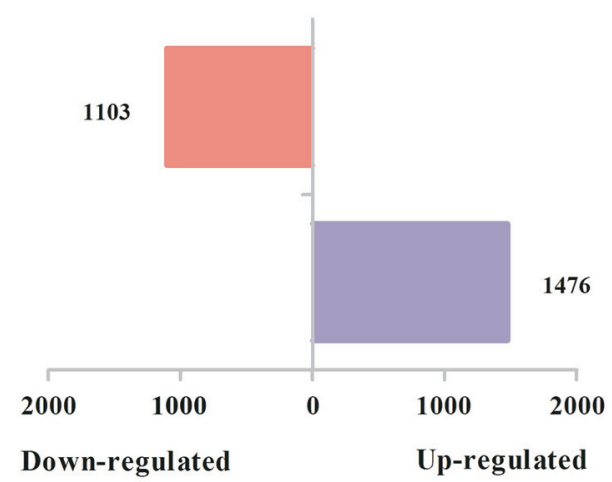

Figure 5 - Differential expression gene analysis of $D$. schraderiana transcriptome. (A) Tissue-specific expressed unigenes. FS: flowers-specific; LS: leaves-specific; $(\mathrm{F}+\mathrm{L})$ : unigenes differentially expressed in common between flowers and leaves. (B) The number of significantly up- and down-regulated unigenes in leaves compared to flowers.

In the GO enrichment analysis, the corrected $p$-value of the enriched function should be below 0.05 (Westfall and Young, 1989). Highly enriched DEGs were found as involved in the reductive pentose-phosphate cycle (83.33\%), photosynthesis, dark reaction (58.82\%), the DNA bending complex (47.22\%), nucleosome (47.22\%), and protein heterodimerization activity $(30.16 \%)$ between flowers and leaves (Figure S2). A further study of DEGs focused on the 
search for significantly enriched biochemical pathways in the KEGG database. Between flowers and leaves, the most significant ( $p$-value $<0.05$ ) enriched pathway was related to metabolisms, such as glyoxylate and dicarboxylate metabolism, photosynthesis, and carbon fixation in photosynthetic organisms (Figure S3).

\section{Transcription Factor (TF) Identification}

The biological characteristics corresponding to spatial, temporal, and environmental stimuli are regulated at the transcriptional level via transcription factors, which have been investigated intensively (Yang et al., 2012). In our study, 2,156 unique unigenes with RPKM values were shown to belonged to 97 plant-specific and plant-non-specific transcription factor families (Table S5). Top 10 transcription factor families are shown in Figure 6. The $\mathrm{C} 2 \mathrm{H} 2$ transcription factor family had the highest number of members (385) among all unigenes, followed by WD40-like (239), PHD (103), MYB-HB-like (99), and bHLH (93). Among the identified unigenes, we found that 255 unigenes distributed in 55 transcription factor families exhibited significant differential expression levels. Most significant differentially expressed unigenes (26) represented in $\mathrm{C} 2 \mathrm{H} 2$ transcription factor family, which acts as trans-regulators of gene expression in cellular processes such as differentiation and development (Razin et al., 2012).

\section{Discussion}

Before this study, no nucleotide sequence from $D$. schraderiana was available in public databases, which limited research on molecular mechanisms of secondary metabolites biosynthesis, physiological adaptation, growth, etc. Nowadays, an increasing number of studies have proved that high-throughput sequence and de novo assembly could be an effective technique for non-model plants to identify novel genes and produce massive sequences at a low cost. (Li et al., 2016; Moraortiz et al., 2016).

Here, an Illumina Hiseq4000 sequencing platform was employed to sequence RNA from flower and leaf tissues, providing the first transcriptome information for $D$. schraderiana, which can now be extensively employed in research for novel gene discoveries, comparative genomics, functional genomics, and phylogenetics. In this study, we have obtained 40,142 unigenes with a mean length of $873.13 \mathrm{bp}$, suggesting that our assembly has high quality according to the criterion for evaluating the accuracy of an assembly. The unigenes were matched with unique known proteins in public databases to predict the potential functions. As a result, 59.45\% were successfully annotated, suggesting their relatively conserved functions. The non-annotated unigenes may possess poorly conserved regions (Hou et al., 2011).

Similarity analyses in $\mathrm{Nr}$ database indicated that the unigenes of $D$. schraderiana had the highest homologies in cDNA sequences of Vitis vinifera (13.93\%), Theobroma cacao (7.73\%), and Populus trichocarpa (4.97\%). According to the Angiosperm Phylogeny Group III (APG III), $V$. vinifera, T. cacao, and P. trichocarpa all belong to Dicotyledoneae, as is also the case with D. schraderiana. Furthermore, reference genome sequences of $V$. vinifera, $T$. cacao, and $P$. trichocarpa are available in the public databases (Jaillon et al., 2007; Wullschleger et al., 2013; Maximova et al., 2014). Therefore, D. schraderiana and these three plant species showed similar genome sequences due to their relatively close relationship.

With respect to the KEGG analysis, D. schraderiana unigenes were mainly related to metabolic pathways $(2,994$ transcripts) and biosynthesis of secondary metabolites (1,417 transcripts). Additionally, the transcriptomic data from flower and leaf tissues showed that 1,476 unigenes exhibited up-regulation, 1,103 unigenes were down-regulated

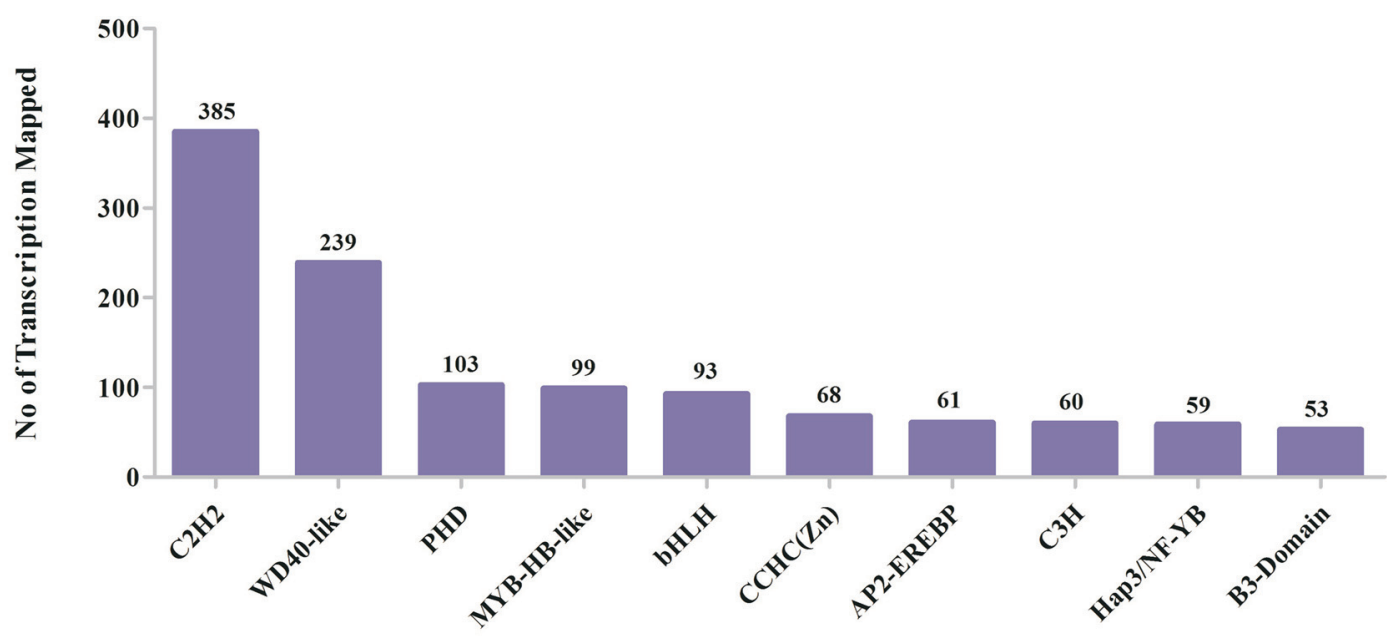

Transcription Factor Family

Figure 6 - Top 10 transcription factor families. 
in leaves compared to flowers, and 234 and 146 unigenes were specific to flower and leaf tissues separately. In both the GO and KEGG enrichment analyses, photosynthesis and energy metabolism were enriched, which is likely due to the leaves vital function of photosynthesis performed by chlorophyll. Besides, 2,156 unique unigenes belonged to 97 transcription factor families.

Based on the KEGG analysis, 28 unigenes related to terpenoid backbone biosynthesis of $D$. schraderiana were identified, involving all enzymes in the MVA and MEP pathways, except for 1-hydroxy-2-methyl-2-(E)-butenyl4-diphosphate reductase (HDR) (Table S6). Additionally, 10 unigenes were predicted to encode two known monoterpene synthases, including (3S)-linalool synthase and (+)-neomenthol dehydrogenase (Table S7). Eight other unigenes were annotated to encode three enzymes participating in sesquiterpene biosynthesis, including valencene/7-epi-alpha-selinene synthase, (-)-germacrene D synthase, and $\mathrm{NAD}^{+}$-dependent farnesol dehydrogenase. The expression levels of these unigenes and their FPKM values were identified and estimated. Unigene c12686_g1 annotated to $(+)$-neomenthol dehydrogenase and unigene c7041_g2 annotated to (-)-germacrene D synthase showed significant differential expression between flowers and leaves (Table S7). And unigene c12686_g1 showed a higher RPKM value in leaf tissue compared to flower tissue, while unigene c7041_g2 had high abundance in flowers. Additional studies involving intensive molecular and proteomic analyses should now be carried out to validate these gene function predictions.

\section{Conclusion}

D. schraderiana is a potential medicinal plant that can adapt to alpine hypoxia and high ambient ultraviolet radiation. In this study, our transcriptome analysis generated 40,142 unigenes, of which $59.45 \%$ were aligned to sequences in databases. This first comprehensive transcriptome analysis markedly expands our understanding of molecular mechanism in $D$. schraderiana. It also provides a large number of candidate genes potentially involved in the biosynthesis of secondary metabolites and could be used in research on the adaptation to the extreme environment of the Qinghai-Tibet Plateau.

\section{Conflict of interests}

The authors declare that they have no competing interests.

\section{Author contributions}

SF conceived and designed the experiments, analyzed the data, prepared figures and/or tables, authored or reviewed drafts of the paper, approved the final draft; ML conceived and designed the experiments, performed the experiments, contributed reagents/materials/analysis tools, approved the final draft; YZ conceived and designed the experiments, analyzed the data, authored or reviewed drafts of the paper, approved the final draft; ZD prepared figures and/or tables, authored or reviewed drafts of the paper, approved the final draft; JS prepared figures and/or tables, authored or reviewed drafts of the paper, approved the final draft; DH prepared figures and/or tables, authored or reviewed drafts of the paper, approved thefinal draft.

\section{References}

Benjamini Y and Hochberg Y (1995) Controlling the false discovery rate - a practical and powerful approach to multiple testing. J R Stat Soc 57:289-300.

Camacho C, Coulouris G, Avagyan V, Ning M, Papadopoulos J, Bealer K and Madden TL (2009) BLAST+: Architecture and applications. BMC Bioinformatics 10:421-429.

Cheng AX, Lou YG, Mao YB, Lu S, Wang LJ and Chen XY (2007) Plant terpenoids: Biosynthesis and ecological functions. J Integr Plant Biol 49:179-186.

Chou WC, Lin SS, Yeh SD, Li SL, Peng YC, Fan YH and Chen TC (2017) Characterization of the genome of a phylogenetically distinct tospovirus and its interactions with the local lesion-induced host Chenopodium quinoa by whole-transcriptome analyses. PLoS One 12: 0182425.

Cock PJ, Fields CJ, Goto N, Heuer ML and Rice PM (2009) The Sanger FASTQ file format for sequences with quality scores, and the Solexa/Illumina FASTQ variants. Nucleic Acids Res 38:1767-1771.

Crawford JE, Guelbeogo WM, Sanou A, Traoré A, Vernick KD, Sagnon NF and Lazzaro BP (2010) De novo transcriptome sequencing in Anopheles funestus using Illumina RNA-Seq technology. PLoS One 5:e14202.

Dai X, Sinharoy S, Udvardi M and Zhao PX (2013) PlantTFcat: An online plant transcription factor and transcriptional regulator categorization and analysis tool. BMC Bioinformatics 14:321-327.

Deng Y, Jianqi LI, Songfeng WU, Zhu Y, Chen Y and Fuchu HE (2006) Integrated $\mathrm{nr}$ database in protein annotation system and its localization. Computer Engineering 32:71-72.

Erlich Y, Mitra PP, Delabastide M, Mccombie WR and Hannon GJ (2008) Alta-Cyclic: A self-optimizing base caller for next-generation sequencing. Nat Methods 5:679-682.

Gershenzon J (2009) Monoterpene and sesquiterpene synthases and the origin of terpene skeletal diversity in plants. Phytochemistry 69:1621-1637.

Goodwin TW (1979) Biosynthesis of terpenoids. Plant Biol 30:369-404.

Grabherr MG, Haas BJ, Yassour M, Levin JZ, Thompson DA, Amit I, Adiconis X, Fan L, Raychowdhury R and Zeng Q (2011) Full-length transcriptome assembly from RNA-Seq data without a reference genome. Nat Biotechnol 29:644652.

Han XJ, Wang YD, Chen YC, Lin LY and Wu QK (2013) Transcriptome sequencing and expression analysis of terpenoid biosynthesis genes in Litsea cubeba. PLoS One 8:e76890.

Harris MA (2004) The Gene Ontology (GO) database and informatics resource. Nucleic Acids Res 32:D258-D261.

Hou R, Bao Z, Wang S, Su H, Li Y, Du H, Hu J, Wang S and Hu X (2011) Transcriptome sequencing and de novo analysis for 
Yesso scallop (Patinopecten yessoensis) using 454 GS FLX. PLoS One 6:e21560.

Jaillon O, Aury JM, Noel B, Policriti A, Clepet C, Casagrande A, Choisne N, Aubourg S, Vitulo N, Jubin C et al. (2007) The grapevine genome sequence suggests ancestral hexaploidization in major angiosperm phyla. Nature 449:463.

Kanehisa M, Goto S, Kawashima S, Okuno Y and Hattori M (2004) The KEGG resource for deciphering the genome. Nucleic Acids Res 32:D277-D280.

Langmead B and Salzberg SL (2012) Fast gapped-read alignment with Bowtie 2. Nat Methods 9:357-359.

Lei M, He H, Zhang P, Yao Y, Zhao Q, Zhang Y and La D (2015a) Study on the Extraction of Chenopodium foetidum essential oil and its inhibition against insect activity. J Anhui Agric Sci 43:64-66.

Lei M, Xue J, Hao D, Shi M, Yao Y, Zhang Y and La D (2015b) Study on incense bacteriostatic of Chenopodium foetidum powder. Tibet Sci Technol 12:63-64.

Li B and Dewey CN (2011) RSEM: Accurate transcript quantification from RNA-Seq data with or without a reference genome. BMC Bioinformatics 12:323-339.

Li J, Zheng W, Long D, Ding L, Gong A, Xiao C, Jiang W, Liu X, Zhou T and Huang L (2016) Correction: De novo sequencing and assembly analysis of the Pseudostellaria heterophylla transcriptome. Plos One 11:e0164235.

Liu Y (2013) Study on quantitative ecology of vegetation alongside the north branch of Wanjiazhai Yellow River diversion project. Shanxi University, Taiyuan.

Liu Z (2015) Faming Zhuanli Shenqing Gongkai Shuomingshu. CN104782667A. China.

Maximova SN, Florez S, Shen X, Niemenak N, Zhang Y, Curtis W and Guiltinan MJ (2014) Genome-wide analysis reveals divergent patterns of gene expression during zygotic and somatic embryo maturation of Theobroma cacao L., the chocolate tree. BMC Plant Biol 14:185.

Moraortiz M, Swain MT, Vickers MJ, Hegarty MJ, Kelly R, Smith LMJ and Skøt L (2016) De novo transcriptome assembly for gene identification, analysis, annotation, and molecular marker discovery in Onobrychis viciifolia. BMC Genomics 17:756-769.

Mortazavi A, Williams BA, Mccue K, Schaeffer L and Wold B (2008) Mapping and quantifying mammalian transcriptomes by RNA-Seq. Nat Methods 5:621-628.

Razin SV, Borunova VV, Maksimenko OG and Kantidze OL (2012) Cys2His2 zinc finger protein family: Classification, functions, and major members. Biochemistry (Mosc.) 77:217-226.

Robinson MD, Mccarthy DJ and Smyth GK (2010) edgeR: A Bioconductor package for differential expression analysis of digital gene expression data. Bioinformatics 26:139-140.

Rohdich F, Eisenreich W, Wungsintaweekul J, Hecht S, Schuhr CA and Bacher A (2001) Biosynthesis of terpenoids. Eur J Biochem 268:3190-3197.
Shi MF (2015) Study on extraction, composition analysis and antibacterial activity of Chenopodium foetidum essential oil. M. Sc. Thesis, Xizang University, Tibet.

Westfall PH and Young SS (1989) P value adjustments for multiple tests in multivariate binomial models. J Am Stat Assoc 84:780-786.

Wullschleger SD, Weston DJ, Difazio SP and Tuskan GA (2013) Revisiting the sequencing of the first tree genome: Populus trichocarpa. Tree Physiol 33:357-364.

Xie C, Mao X, Huang J, Ding Y, Wu J, Dong S, Kong L, Gao G, Li CY and Wei L (2011) KOBAS 2.0: A web server for annotation and identification of enriched pathways and diseases. Nucleic Acids Res 39:316-322.

Xie Z (1996) National Compendium of Chinese Herbal Medicine. People 's Health Publishing House, Beijing, 1050 p.

Yang CQ, Fang X, Wu XM, Mao YB, Wang LJ and Chen XY (2012) Transcriptional regulation of plant secondary metabolism. J Integr Plant Biol 54:703-712.

Young MD, Wakefield MJ, Smyth GK and Alicia O (2010) Gene ontology analysis for RNA-seq: Accounting for selection bias. Genome Biol 11:R14.

Zhang YF, Li GL, Wang XF, Sun YQ and Zhang SY (2017) Transcriptomic profiling of taproot growth and sucrose accumulation in sugar beet (Beta vulgaris L.) at different developmental stages. PLoS One 12:e175454.

\section{Supplementary material}

The following online material is available for this study:

Table S1 - Quality of sequencing.

Table S2 - KEGG classification for unigenes of D. schraderiana.

Table S3 - Unigenes (2579) with detectable expression level.

Table S4 - KEGG annotation of DEGs

Table S5 - List of predicted transcription factors.

Table St - Unigenes involved in terpenoid backbone biosynthesis.

Tahle Si - Unigenes annotated to mono-TPS and sesquiTPS in D. schraderiana.

Figure S I - Pathway assignment based on the KEGG database.

Figure S2 - Flowers vs Leaves.DE.list.GO.enrichment.details.

Figure S3 - Flowers vs Leaves.DE.list.KEGG.enrichment.details.

Associate Editor: Houtan Noushmehr

License information: This is an open-access article distributed under the terms of the Creative Commons Attribution License (type CC-BY), which permits unrestricted use, distribution and reproduction in any medium, provided the original article is properly cited. 